Focussed on "Crack Paths"

\title{
Determination of dissipated Energy in Fatigue Crack Propagation Experiments with Lock-In Thermography
}

\author{
Jürgen Bär, Ralf Urbanek \\ University of the Bundeswebr München, Germany \\ juergen.baer@unibw.de,bttp://orcid.org/0000-0003-1157-0052 \\ Ralf.urbanek@unibw.de
}

\begin{abstract}
Lock-In thermography can be used to investigate elastic stresses and dissipative effects in dynamic processes like crack propagation. The evaluation normally is performed with a Discrete Fourier Transformation resulting in E- and D-Amplitude and phase images. The E-Amplitude images give information about the stress distribution, the D-Amplitude is connected with dissipated energies. The observed changes in the E-amplitude values can be attributed to a change in the stress state at the crack tip due to bending of the specimen caused by the propagating crack. In the crack propagation experiments the maximum value of the D-Mode in the area in front of the crack tip was found to be constant. The appearance of higher harmonic modes in the evaluation raises the question if the Discrete Fourier Transformation is the appropriate method for a quantitative evaluation of dissipative effects. Experiments performed on flat specimens show that the temperature change due to dissipative effects could not be described with a sine wave with the double loading frequency. Therefore, a quantitative determination of dissipated energies using the Discrete Fourier Transformation is impossible. For a quantitative determination of dissipated energies a new evaluation method has to be developed.
\end{abstract}

KEYWORDS. Aluminum alloy; Crack propagation; Dissipated energy; Fatigue; Thermography.

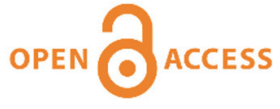

Citation: Bär, J., Urbanek, R., Determination of dissipated Energy in Fatigue Crack Propagation Experiments with Lock-In Thermography, Frattura ed Integrità Strutturale, 48 (2019) 563-570.

Received: 26.11 .2018

Accepted: 20.12.2018

Published: 01.04.2019

Copyright: (C) 2019 This is an open access article under the terms of the CC-BY 4.0, which permits unrestricted use, distribution, and reproduction in any medium, provided the original author and source are credited.

\section{INTRODUCTION}

$\mathrm{T}$ hermographic methods are widely used to determine the temperature increase during a fatigue experiment. Different methods were developed to gather information about the fatigue limit and the fatigue lifetime out of the measured temperature changes [1-4]. In case of a propagating crack, the local conditions are changing very fast, therefore a simple temperature measurement is not sufficient. In this case, the Lock-In thermography offers the possibility to gather information about elastic stress fields and dissipated energies. In case of the thermoelastic stimulated Lock-In Thermography, a cyclic change of the temperature is caused by an alternating load according to the thermoelastic effect [5]. 
For a specimen loaded at the temperature $T_{m}$ with a stress amplitude $\sigma_{a}$ and a frequency $f_{L}$ the temperature at the time $t$ is given by Eq. (1):

$$
T(t)=-T_{m} \cdot K_{0} \cdot \sigma_{a} \cdot \sin \left(2 \pi f_{L} \cdot t\right)=-T_{m} \cdot \frac{\alpha}{\rho \cdot c_{p}} \cdot \sigma_{a} \cdot \sin \left(2 \pi f_{L} \cdot t\right)
$$

The parameter $\mathrm{K}_{0}$ represents the thermoelastic constant, which can be calculated from the coefficient of thermal expansion $\alpha$, the density $\rho$ and the specific heat capacity $c_{p}$ of the material. To enhance the thermal emissivity of the metallic surface, the specimens have to be painted. The emissivity of the coating has to be taken into account for a correct determination of elastic stresses out of the measured temperature amplitude. When the thermal emissivity of the coating is unknown, a thermoelastic constant $\mathrm{K}_{\mathrm{c}}$ including the emissivity of the paint can be determined by calibrating the system with defined stress amplitudes [6]. In this case, $K_{c}$ replaces the constant $K_{0}$ in Eq. (1). With the Thermoelastic Stress Analysis (TSA) it is possible to measure local stress fields of cyclic loaded components using Eq. (1). Unfortunately, only the stress amplitude and not the maximum stress can be determined.

Several authors [7-10] have shown that in case of plastic deformation a second mode coupled with the double loading frequency appears. They assigned it to dissipative energies and integrated that part as the so-called D-Mode into the evaluation. The corresponding evaluation is based on an incomplete Discrete Fourier Transformation (DFT) and can be written as:

$$
T(t)=\underbrace{T_{m}}_{\text {average Temperature }}+\underbrace{T_{E} \cdot \sin \left(2 \pi f_{L} \cdot t+\varphi_{E}\right)}_{E-\text { Mode }}+\underbrace{T_{D} \cdot \sin \left(2 \cdot 2 \pi f_{L} \cdot t+\varphi_{D}\right)}_{D \text {-Mode }}+\underbrace{\Phi(t)}_{\text {Noise }}
$$

In this evaluation, the temperature signal is dissected into a mean temperature $\mathrm{T}_{\mathrm{m}}$, a thermoelastic part coupled with the loading frequency (E-Mode) and a dissipative part, the so-called D-Mode, coupled to the double loading frequency. The noise $\Phi(t)$ of the temperature signal can be eliminated with this dissection of the temperature signal. This evaluation generates an image for the E- and D-Amplitude $\left(T_{E}\right.$ and $\left.T_{D}\right)$, the E- and D-Phase $\left(\varphi_{E}\right.$ and $\left.\varphi_{D}\right)$ and the average temperature $\mathrm{T}_{\mathrm{m}}$, respectively. Due to the limited recording frequency, $\mathrm{f}_{\mathrm{R}}$, of thermographic cameras, normally several cycles were used for the evaluation. Therefore, it is necessary to use a recording frequency $f_{R}$ that is not an even multiple of the testing frequency $f_{L}$. During the measurement, the average temperature $T_{m}$ is assumed to be constant. A changing mean temperature is considered in the approach published by de Finis et al [11].

Urbanek and Bär $[12,13]$ extended the approach to higher harmonic frequencies up to the Nyquist frequency, resulting in additional D1 and D2 modes:

$$
T(t)=\underbrace{T_{m}}_{\text {average temperature }}+\underbrace{T_{E} \cdot e^{2 \pi i\left(f_{L} \cdot t+\varphi_{E}\right)}}_{\begin{array}{c}
\text { thermo-elastic } \\
(E-\text { Mode })
\end{array}}+\underbrace{T_{D} \cdot e^{2 \pi i\left(2 f_{L} \cdot t+\varphi_{D}\right)}}_{\begin{array}{c}
\text { dissipative } 2 f_{L}-\text { part } \\
(D-\text { Mode })
\end{array}}+\underbrace{\sum_{k=1}^{N_{N y q u i s t}} T_{D_{k}} \cdot e^{2 \pi i\left((k+2) f_{L} \cdot t+\varphi_{k}\right)}}_{\begin{array}{c}
\text { higher harmonics } \\
\left(D_{1}, D_{2}, \ldots, \ldots-M o d e\right)
\end{array}}+\underbrace{\Phi(t)}_{\text {Noise }}
$$

In all approaches, the noise of the temperature measurement is suppressed, leading to a good temperature resolution of about $1.6 \mathrm{mK}$ in the resulting amplitude images $[12,13]$. The Lock-In Thermography is suitable for the investigation of dynamic processes, for example in crack propagation experiments.

\section{EXPERIMENTAL DETAILS}

\section{Crack Propagation Experiments}

7 he experiments were carried out on single edge notched specimens with a size of $80 \mathrm{~mm} \times 12 \mathrm{~mm} \times 2.85 \mathrm{~mm}$ machined from EN AW 7475-T761 clad sheet material. A U-type notch with a notch radius of $0.5 \mathrm{~mm}$ and a width and a depth of $1 \mathrm{~mm}$ was milled into one side of each specimen. The fatigue crack propagation experiments were performed under fully reversed loading conditions at a frequency of $20 \mathrm{~Hz}$ in a special equipped servohydraulic testing machine with fixed grips to minimize bending forces. The direct measurement of the potential drop and the subsequent online calculation of the crack length enables experiments under stress intensity controlled conditions. Bär and Volpp [14] give a detailed description of the testing equipment. 
The experiments were undertaken with constant stress intensities $\mathrm{K}_{\max }=$ const. and $\Delta \mathrm{K}=$ const.. The crack initiation and the first part of the crack propagation experiment were performed with a constant force amplitude until the desired stress intensity, due to the increasing crack length, was achieved. Henceforward, the experiments were performed with constant stress intensities, $\mathrm{K}_{\max }=5 ; 7.5$ and $10 \mathrm{MPa} \sqrt{m}$, until a maximum crack length of $8 \mathrm{~mm}$, respectively. For each stress intensity level, five specimens have been tested.

\section{Lock-In Thermography}

The fatigue crack propagation experiments were accompanied by thermographic measurements performed with a CEDIP Titanium HD 560 camera. During the crack propagation experiments, the camera automatically recorded sequences of 990 frames with a framerate of $99 \mathrm{~Hz}$ in defined intervals of 1 min. Each frame consists of 640 x 512 pixel with a spatial resolution of $29 \mu \mathrm{m} /$ pixel. For the Lock-In analysis the load, delivered from the control electronics of the testing machine in form of an analogue signal, was recorded by the camera in form of a 14-bit value. To achieve a high and equal emissivity, the surface of the specimens was grinded and coated with a layer of black paint with a thickness of about $20 \mu \mathrm{m}$.

The Lock-In evaluation of the thermographic measurements was undertaken with a self-developed MatLab program, including a motion compensation algorithm to compensate the rigid body movement of the specimen during a sequence [11]. The Lock-In evaluation was performed for each pixel of the recorded frames using a Discrete Fourier Transformation according to Eq.(3).
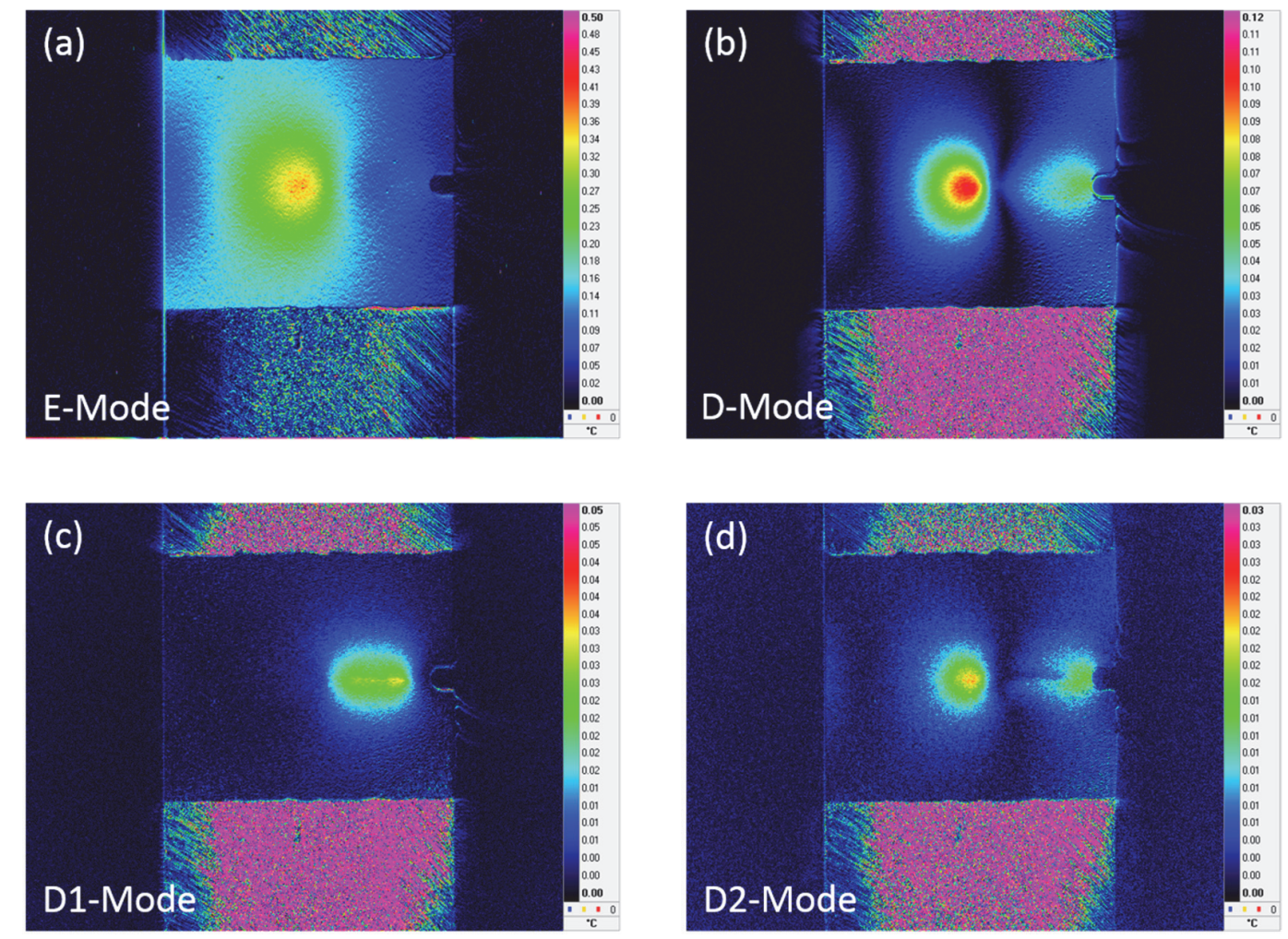

Figure 1: Resulting amplitude images of the DFT according to Eq. (3): The E-Mode (a) is coupled with the loading frequency, the D-Mode (b), the D1-Mode (c) and the D2-Mode represent the first, second and third harmonic.

For each analyzed sequence of 990 frames, the evaluation provides an image for the average temperature $T_{m}$, as well as, amplitude and phase images for the E-, D-, D1- and D2-Mode, respectively. The DFT evaluation reduces the noise down to a value of $1.6 \mathrm{mK}$. An example for the resulting images for a specimen loaded with $\mathrm{K}_{\max }=10 \mathrm{MPa} \sqrt{\mathrm{m}}$ and a crack length of $\mathrm{a}=7.1 \mathrm{~mm}$ is shown in Fig. 1 .

To compare the results of the thermographic measurements obtained in different experiments, the results were quantified by determining the arithmetic mean of all amplitude values and the maximum value within defined areas. For a detailed examination, a separate evaluation was undertaken for the crack tip and the crack flanks (Fig. 2). At the crack tip an area of $73 \times 73$ pixels $(2.05 \times 2.05 \mathrm{~mm})$ was located axially symmetric to the notch root. This area was shifted automatically in the 
evaluation program with the propagating crack perpendicular to the loading direction until the mean value of the Eamplitude within the square reaches a maximum (red square in Fig 1). With this routine, it was assured that the evaluation area covers the highest deformed area in front of the crack tip. To quantify the thermographic data along the crack flanks, all pixels in a stripe with a height of 73 pixels between the square at the crack tip and the notch root were analyzed (yellow square in Fig. 2).

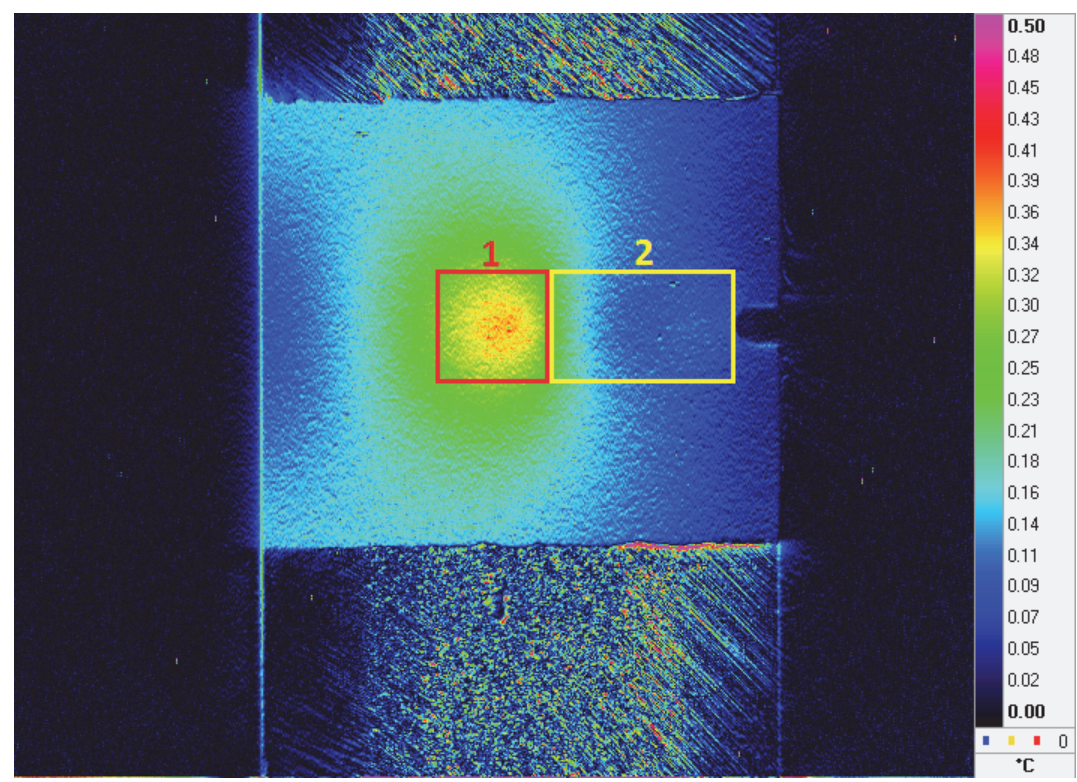

Figure 2: Areas for the evaluation of the Lock-In measurements.

\section{RESULTS}

I

n Fig. 3 the mean and maximum amplitude values of the E- and D-Mode in the area at the crack tip are shown. The general level of the E- and D-Amplitude values are increasing with the applied stress intensity. The values of the DAmplitude are about 5 times lower compared to the E-Amplitude. The mean and maximum E-Amplitude values decrease with increasing crack length, which can be attributed to the decrease in the applied force. In case of the mean values of the E- and D-Amplitude all specimen tested with the same stress intensity show the same run with a low scatter. The mean D-Amplitude shows for all investigated stress intensities an increase, up to a crack length of about $6 \mathrm{~mm}$. Above a crack length of $6 \mathrm{~mm}$, the values are nearly constant or slightly decrease with the crack length. The maximum D-values show nearly the same values independent of the crack length.

The measured mean values for the D1 and D2-Amplitude are about 5 times lower compared to the D-Amplitude (Fig. 4). For these higher D-Modes, significant differences between specimens tested at the same stress intensity are visible. But the absolute values of these differences are in the same order of magnitude as the calculated noise of about $1.6 \mathrm{mK}$.

The maximum values of the D1 and D2-amplitude are significant higher compared to the mean values. After a decrease up to a crack length of about $3 \mathrm{~mm}$, the D1- and D2-Amplitude values remain constant within a scatter band. In contrast to the maximum values of the D-Amplitude, where the values show a clear dependency of the applied stress intensity, in case of the D1 and D2-Amplitude this dependency is less pronounced.

As in the case of the area at the crack tip, in the area 2 along the crack flanks, the mean E-amplitude values decrease with the crack length, too. These values are not very meaningful, because the size of the area is increasing with the crack length. The D-Amplitude shows an increase up to a crack length of about $4 \mathrm{~mm}$, followed by a slight decrease with increasing crack length. As in case of the E-Amplitude, the influence of the applied stress intensity is evident. The D1- and D2-Amplitude values do not show this clear dependence of the stress intensity. The measured values are nearly constant or just slightly rising. The measured values are in the same order as in case of the area at the crack tip. The measured mean D-Amplitude values are significant smaller compared to the values obtained at the crack tip; the values of the D1- and D2-Amplitude are in the same order. This indicates that more energy is dissipated at the crack tip than along the crack flanks. 

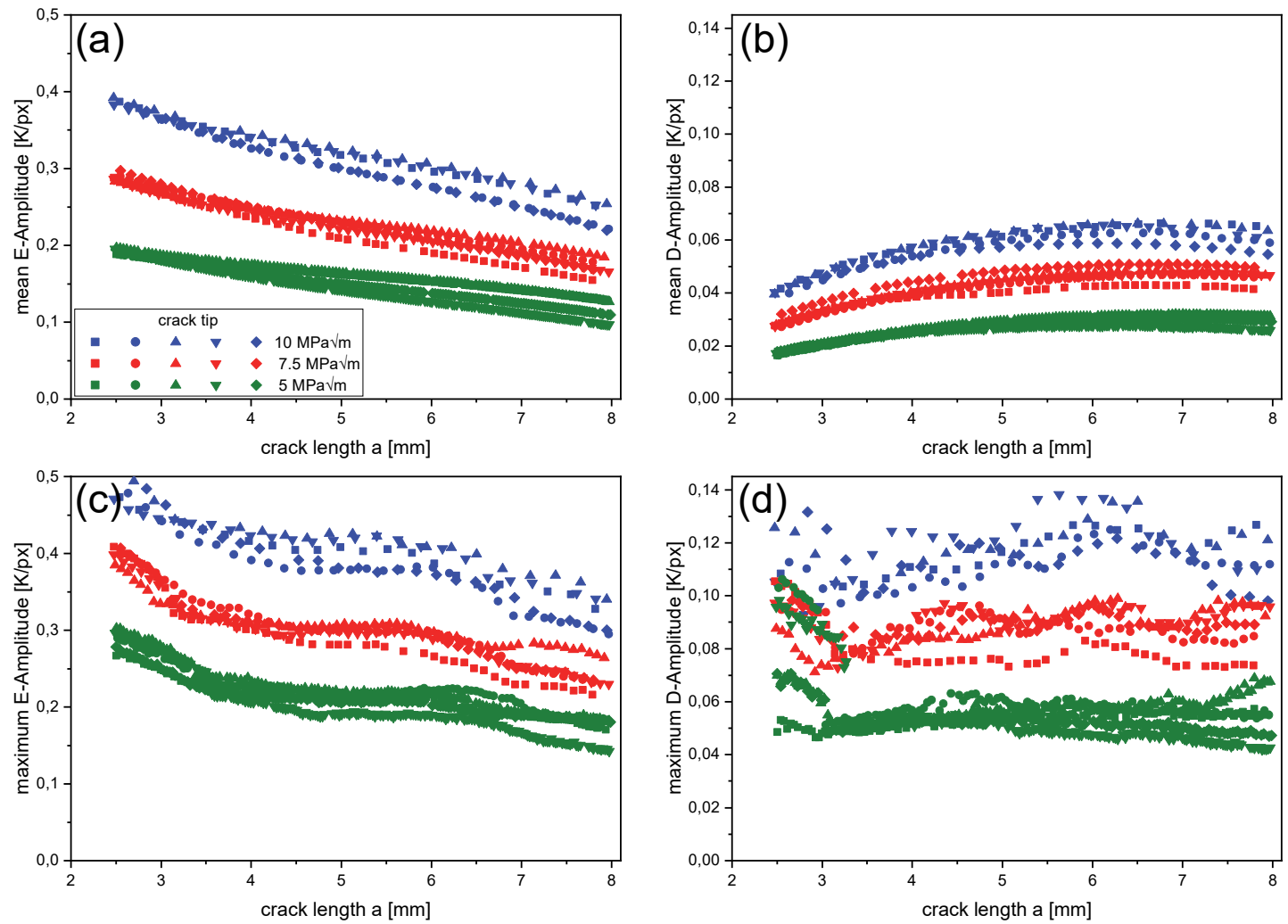

Figure 3: Amplitude values in the area at the crack tip. Mean (a) and maximum (c) values of the E-Mode and mean (b) and maximum (d) values of the D-Mode.
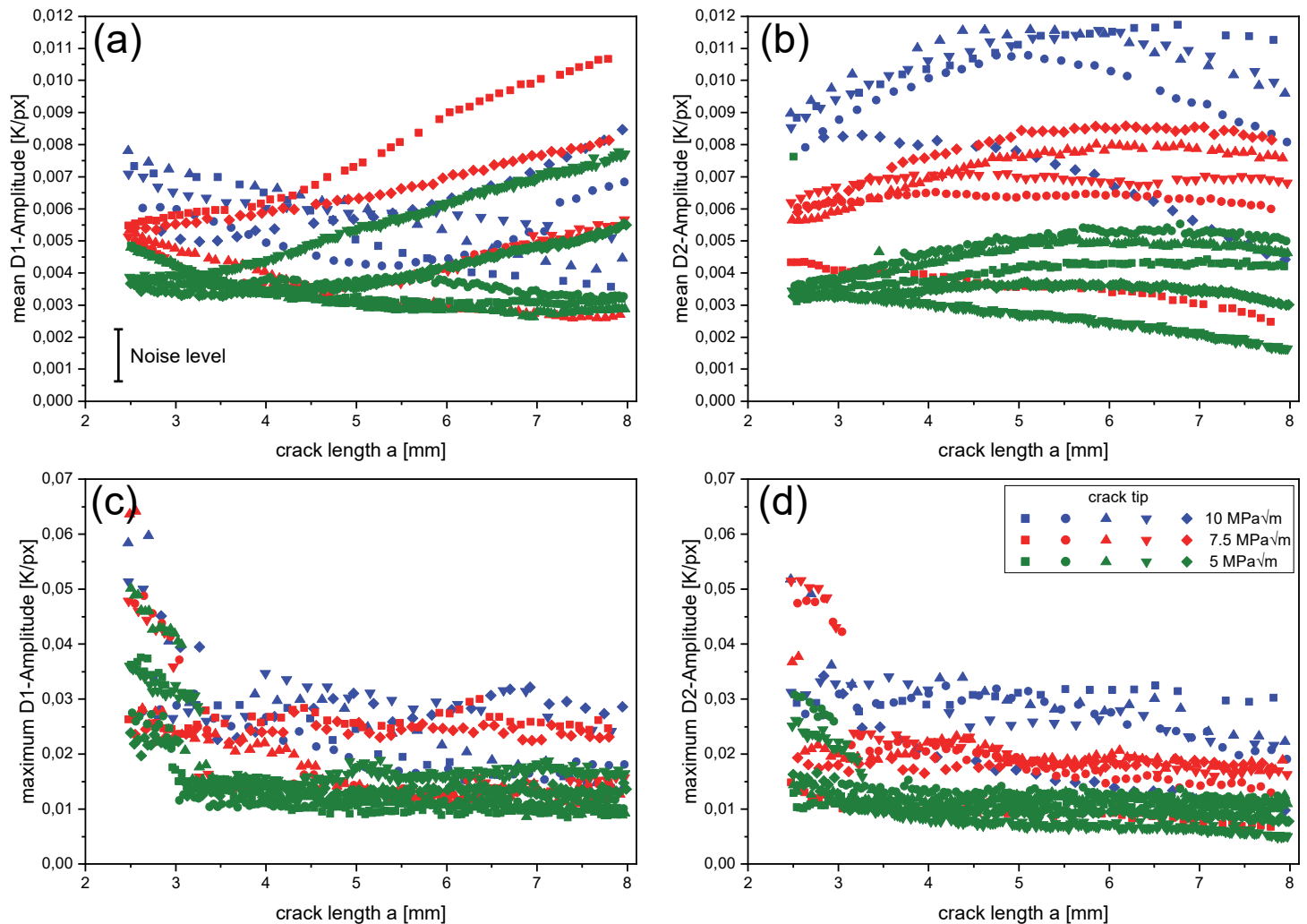

Figure 4: Amplitude values of the higher harmonics in the area at the crack tip. Mean (a) and maximum (c) values of the D1-Mode and mean (b) and maximum (d) values of the D2-Mode. 

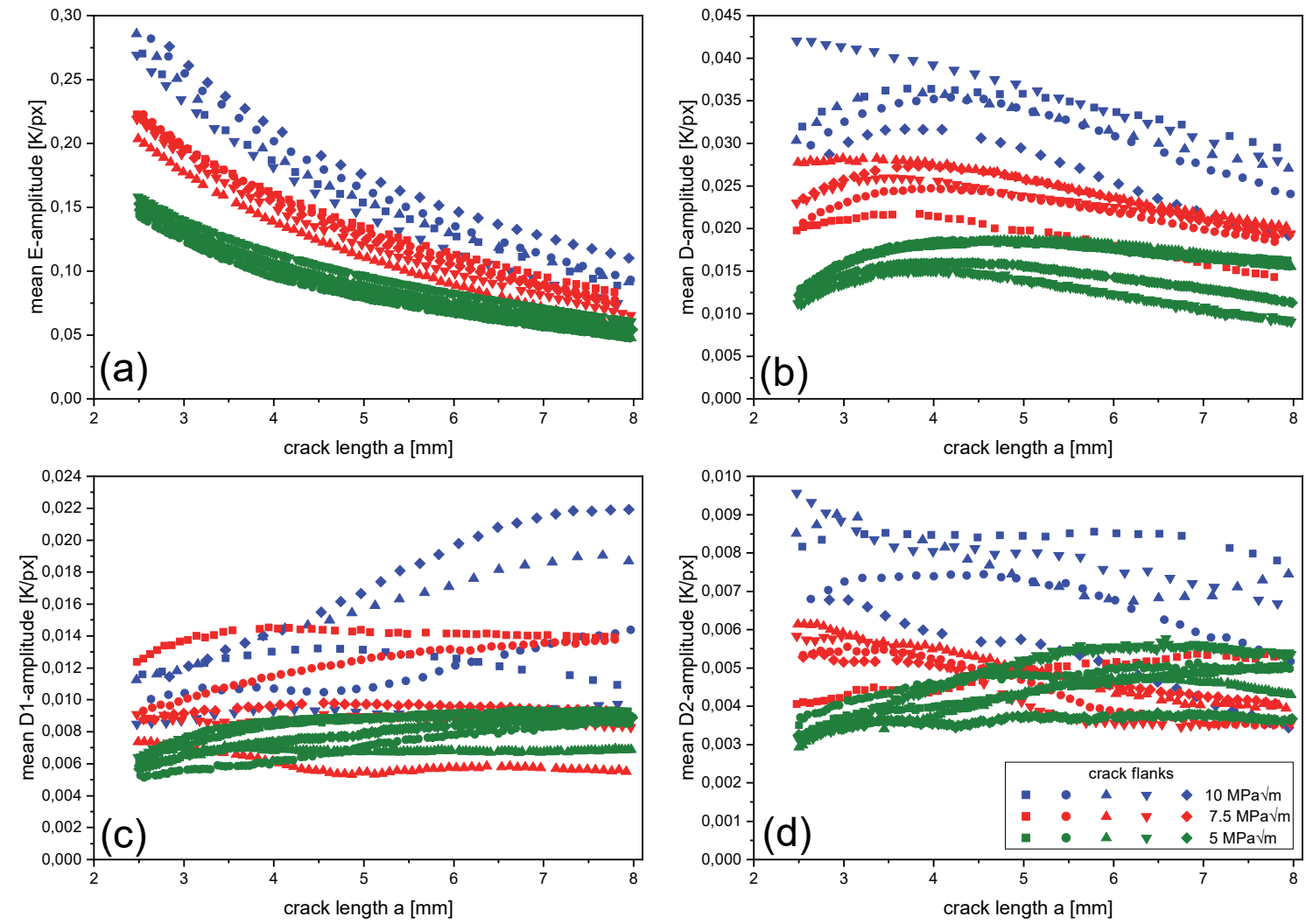

Figure 5: Mean amplitude values of the E-Mode (a), D-Mode (b), D1-Mode (c) and D2-Mode (d) in the area along the crack flanks.

\section{Discussion}

$\mathrm{T}$ he experiments have shown, that the E-Amplitude values decrease with the crack length, although the stress intensity is kept constant. The E-Amplitude values, representing the thermoelastic effect, are coupled with the elastic stresses.

The temperature change can be calculated from the change of the sum of the principal stresses $\sigma_{a}$ by the thermoelastic law (Eq. (1)). The specimen were tested under pure Mode I loading and the parallel guided grips minimize bending forces. Bending forces cannot be avoided completely, especially for long cracks. Fig. 6 shows an E-amplitude image of a specimen loaded with a stress intensity of $10 \mathrm{MPa} \sqrt{m}$ at a crack length of $4.5 \mathrm{~mm}$ (Fig. 6a) and $8 \mathrm{~mm}$ (Fig. 6b), respectively. In case of the short crack, the temperature and therefore the stress between the plastic zone and the border of the specimen is nearly perfect homogenous. In case of the long crack the stress state in front of the crack tip is influenced by the limited specimen width. The influence of limited specimen width is already known in presence of deep notches [13]. This additional bending stress reduces the sum of principal stresses with increasing crack length, which reduces the temperature changes at the crack tip, although the stress intensity in the loading direction is constant. This shows that the DFT evaluation delivers reliable and traceable results for the E-Mode values.

The maximum values of the D-Mode were found to be independent of the crack length. Due to the constant stress intensity in the experiments, this result is well understandable. The D-Amplitude values at the crack tip are considerable higher compared to the amount at the crack flanks where the highest D-Amplitude values are visible near the notch (Fig. 1b). An unsolved problem is the interpretation of the D1- and the D2-Amplitudes. Especially the D1-Amplitude, which is connected with triple loading frequency, is difficult to connect with effects in the material. The evaluation of the thermographic measurements with a DFT according to Eq. (2) or (3) represents a division of the measured signal into a sum of sine waves. After Sakagami [8] Brémond [9] and Shiozawa [10], the dissipative effects can be described with a sine wave with the double loading frequency. To check this assumption, experiments on flat specimens without a notch were undertaken. To determine the thermoelastic constant and the influence of the coating, in a first step a pure elastic loading with a frequency of $5 \mathrm{~Hz}$ was applied. The temperature signal was recorded with a Infratec ImageIR $8300 \mathrm{hp}$ camera with a sampling rate of $1000 \mathrm{~Hz}$ 
and an image size of 160x120 pixel. In a second step, an elastic-plastic loading was applied on the specimen. The recorded mean temperature of all pixels within the image $\Delta \mathrm{T}_{\text {measured }}$ is shown in Fig. $7 \mathrm{a}$ as a green line. The corresponding thermoelastic temperature change $\Delta \mathrm{T}_{\text {elastic }}$ was calculated according to Eq. (1) (red dashed line in Fig. $7 \mathrm{a}$ ) and subtracted from the measured temperature change $\Delta \mathrm{T}_{\text {measured }}$ (green line in Fig. 7a). As a result, the temperature change due to dissipative

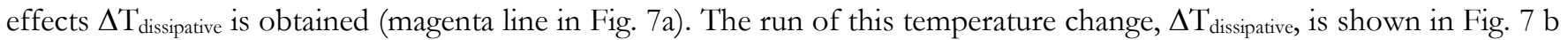
in higher resolution. Due to dissipative effects, a remarkable temperature increase is visible starting when the load maximum is reached. In compression, only a slight increase is visible. These results clearly indicates that the temperature change due to dissipative effects cannot be described by a sine wave with double loading frequency. When a complete DFT according to Eq. (3) is used for the dissection of the temperature signal, amplitude values for the higher harmonic modes (D1 and D2) appear. These higher harmonic modes cannot be assigned to physical effects in the material. The entirety of the higher harmonic modes are just a mathematical description of the deviation of the temperature signal from a pure sine wave (see magenta line in Fig. 7b).
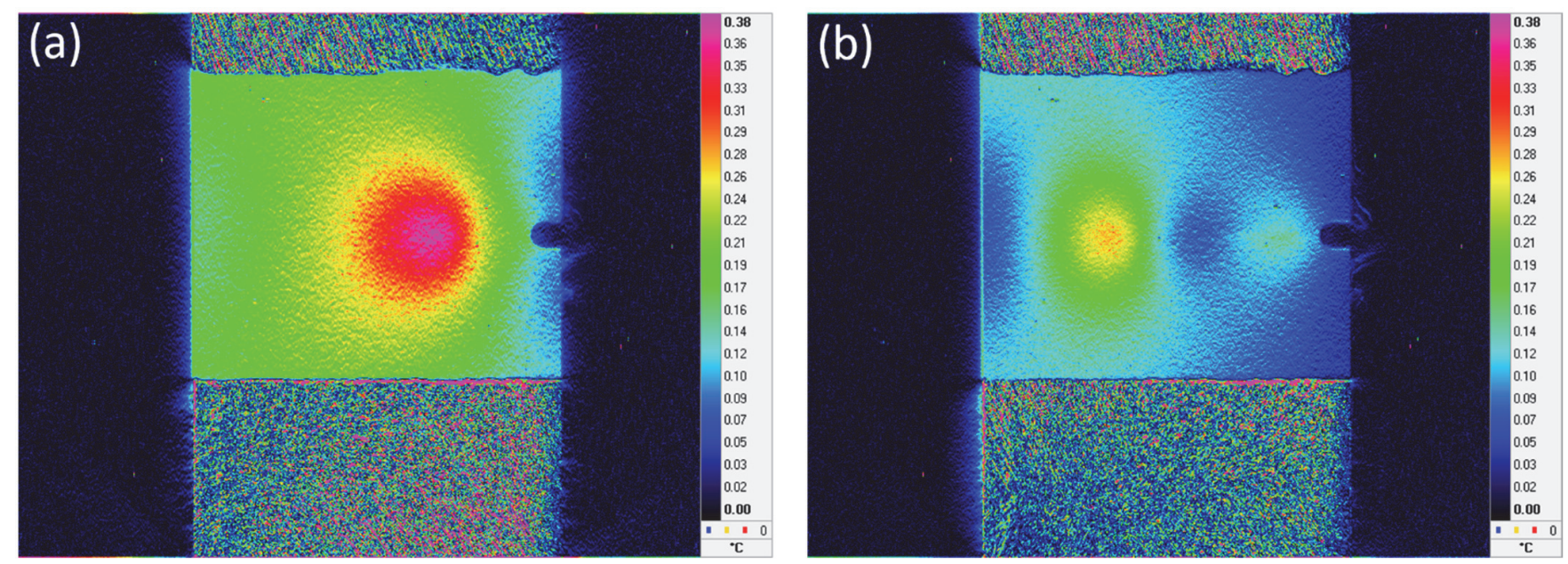

Figure 6: E-Amplitude images of (a) short $(4.5 \mathrm{~mm})$ and (b) long crack $(8 \mathrm{~mm})$.

For a qualitative determination of the dissipated energies, the DFT-approach is sufficient. For a quantitative determination of the temperature change due to dissipative effects and therefore the dissipated energy, a summation of the amplitudes of the D, D1 and D2-Modes may provide an improvement, but for a realistic quantitative description, a new evaluation method has to be developed. Therefore, a detailed analysis of the temperature change during cycling in the elastic-plastic regime is necessary. Without this knowledge the development of a method for a quantitative determination of dissipated energies with the thermoelastic stimulated Lock-In-Thermography is impossible.
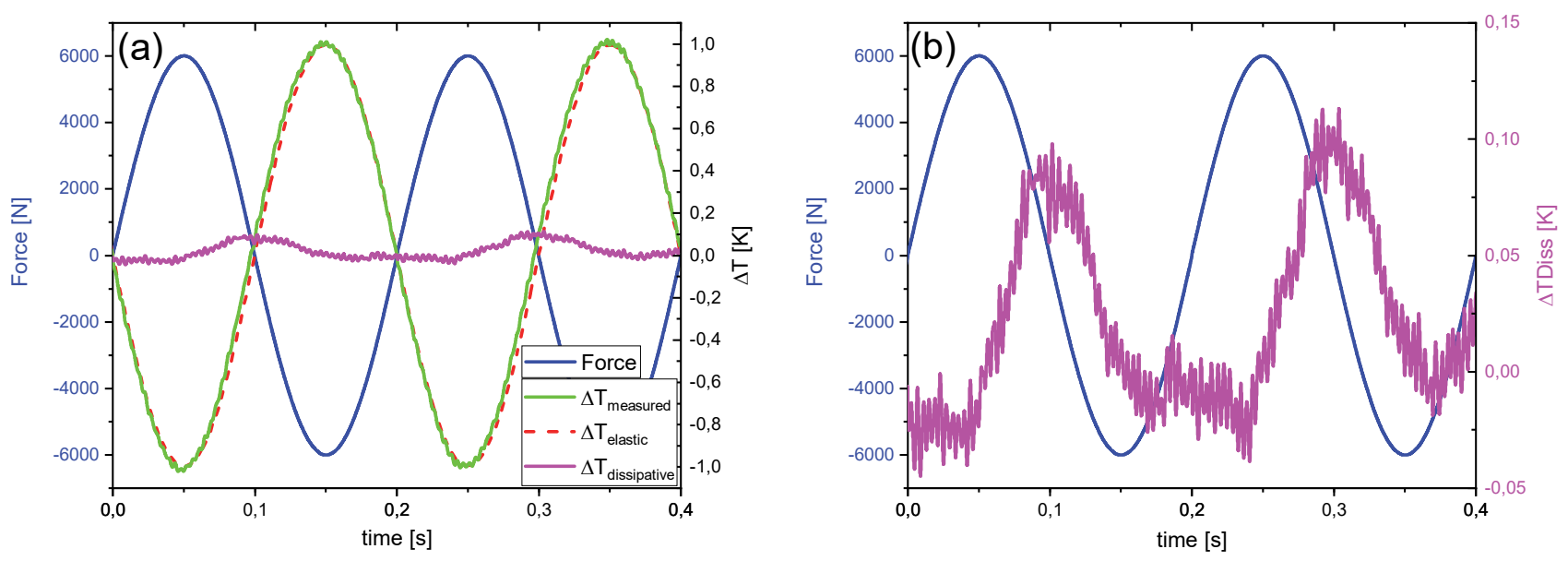

Figure 7: (a) extracting the temperature change due to dissipative effects from the measured temperature change, (b) run of the temperature change due to dissipative effects. 


\section{REFERENCES}

[1] Luong, M.P. (1995). Infrared Thermographic scanning of fatigue in metals. Nuclear Engineering and Design 158, pp. 363-373. DOI: 10.1016/0029-5493(95)01043-H.

[2] La Rosa, G., Risitano, A. (2002). Thermographic methodology for rapid determination of the fatigue limit of materials and mechanical components. International Journal of Fatigue 22, pp. 65-73. DOI: 10.1016/S0142-1123(99)00088-2.

[3] Meneghetti, G. (2007). Analysis of the fatigue strength of a stainless steel based on the energy dissipation. International Journal of Fatigue 29, pp. 81-94. DOI: 10.1016/j.ijfatigue.2006.02.043.

[4] De Finis, R., Palumbo, D., Ancona, F., Galietti, U. (2015). Fatigue limit evaluation of various martensitic stainless steels with new robust thermographic data analysis. International Journal of Fatigue 74, pp. 88-96. DOI: 10.1016/j.ijfatigue.2014.12.010.

[5] Thomson, W. (1853). On the Dynamical Theory of Heat, with numerical results deduced from Joule's equivalent of a Thermal Unit. Transactions of the Royal Society of Edingburgh 20, pp. 261-288.

[6] Urbanek, R., Bär, J. (2017). Lock-In Thermographic Stress Analysis of notched and unnotched specimen under alternating loads. Procedia Structural Integrity 5, pp. 785-792. DOI: 10.1016/j.prostr.2017.07.170.

[7] Enke, N.F., Sandor, B.I. (1988). Cyclic plasticity analysis by differential infrared thermography. Proceedings of the VII International Congress on Experimental Mechanics, pp. 830-835.

[8] Sakagami, T, Kubo, S, Tamura, E., Nishimura, T. (2005). Identification of plastic-zone based on double frequency lockin thermographic temperature measurement. Proceedings of ICF 11, pp. 3751-3756.

[9] Brémond, P. (2007). New developments in Thermo Elastic Stress Analysis by Infrared Thermography. IV PanAmerican Conference for Non Destructive Testing.

[10] Shiozawa, D., Inagawa, T., Washio, T., Sakagami, T. (2016). Fatigue limit estimation of stainless steels with new dissipated energy data analysis. Procedia Structural Integrity 2, pp. 2091-2096. DOI: 10.1016/j.prostr.2016.06.262.

[11] Finis, R. de, Palumbo, D., Galietti, U. (2016). Mechanical Behaviour of Stainless Steels under Dynamic Loading: An Investigation with Thermal Methods. Journal of Imaging 2. DOI: 10.3390/jimaging2040032.

[12] Urbanek, R., Bär, J. (2017). Lock-In Thermographic Stress Analysis of notched and unnotched specimen under alternating loads. Procedia Structural Integrity 5, pp. 785-792. DOI: 10.1016/j.prostr.2017.07.170.

[13] Urbanek, R., Bär, J. (2017). Influence of motion compensation on lock-In thermographic investigations of fatigue crack propagation. Engineering Fracture Mechanics 183, pp. 13-25, DOI: 10.1016/j.engfracmech.2017.03.043.

[14] Bär, J., Volpp, T., Vollautomatische Experimente zur Ermüdungsrissausbreitung, Materialprüfung 43, pp. 242-247. 